\title{
THE TEICHMÜLLER SPACE OF AN ARBITRARY FUCHSIAN GROUP1
}

\author{
BY CLIFFORD J. EARLE
}

Communicated by L. Bers, May 7, 1964

1. Introduction. Let $U$ be the upper half plane. Let $\Sigma$ be the set of quasiconformal self-mappings of $U$ which leave 0,1 , and $\infty$ fixed. The universal Teichmüller space of Bers is the set $T$ of mappings $h: R \rightarrow R$ which are boundary values of mappings in $\Sigma$.

Let $M$ be the open unit ball in $L_{\infty}(U)$. For each $\mu$ in $M$, let $f^{\mu}$ be the unique mapping in $\Sigma$ which satisfies the Beltrami equation

$$
f_{\bar{z}}=\mu f_{z} \text {. }
$$

We map $M$ onto $T$ by sending $\mu$ to the boundary mapping of $f^{\mu}$. T is given the quotient topology induced by the $L_{\infty}$ topology on $M$. The right translations, of the form $h \rightarrow h \circ h_{0}$, are homeomorphisms of $T$.

We shall also associate to each $\mu$ in $M$ a function $\phi^{\mu}$ holomorphic in the lower half plane $U^{*}$. For each $\mu$, let $w^{\mu}$ be the unique quasiconformal mapping of the plane on itself which is conformal in $U^{*}$, satisfies (1) in $U$, and leaves 0,1 , and $\infty$ fixed. $\phi^{\mu}$ is the Schwarzian derivative $\left\{w^{\mu}, z\right\}$ of $w^{\mu}$ in $U^{*}$. By Nehari [3], $\phi^{\mu}$ belongs to the Banach space $B$ of holomorphic functions $\psi$ on $U^{*}$ which satisfy

$$
\|\psi\|=\sup \left|\left(z-z^{*}\right)^{2} \psi(z)\right|<\infty .
$$

It is known [1, pp. 291-292] that $\phi^{\mu}=\phi^{\nu}$ if and only if $f^{\mu}$ and $f^{\nu}$ have the same boundary values. Hence, there is an injection $\theta: T \rightarrow B$ which sends the boundary function of $f^{\mu}$ to $\phi^{\mu}$. We shall write $\theta(T)=\Delta$.

Now let $G$ be a Fuchsian group on $U$; that is, a discontinuous group of conformal self-mappings of $U$, not necessarily finitely generated. The mapping $f$ in $\Sigma$ is compatible with $G$ if $f \circ A \circ f^{-1}$ is conformal for every $A$ in $G$. The Teichmüller space $T(G)$ is the set of $h$ in $T$ which are boundary values of mappings compatible with $G$. The space $B(G)$ of quadratic differentials is the set of $\phi$ in $B$ such that

$$
\phi(A z) A^{\prime}(z)^{2}=\phi(z) \quad \text { for all } A \text { in } G .
$$

Ahlfors proved in [1] that $\Delta$ is open in $B$. Bers [2] proved that $\theta$ maps $T$ homeomorphically on $\Delta$ and maps $T(G)$ onto an open subset of $B(G)$. These results are summed up in the following theorems:

${ }_{1}^{1}$ This research was supported by the National Science Foundation grant NSFGP780. 
THEOREM 1. The mapping $\mu \rightarrow \phi^{\mu}$ is continuous.

ThEOREM 2. The mapping $\mu \rightarrow \phi^{\mu}$ is open.

Theorem 3. $\theta(T(G))$ is an open subset of $B(G)$.

Our purpose here is to give new, more elementary proofs of Theorems 2 and 3 . In particular, we notice that Theorem 3 is a straightforward consequence of Theorems 1 and 2 and the lemma in the next section.

2. The space $D(G)$. For each Fuchsian group $G$, we denote by $D(G)$ the set of $h$ in $T$ such that $h \circ A \circ h^{-1}$ is the boundary function of a conformal self-mapping of $U$ for every $A$ in $G$. Clearly, $T(G)$ is contained in $D(G)$.

LEMMA. $\theta(D(G))=B(G) \cap \Delta$.

Proof. For each $A$ in $G$ and $\phi^{\mu}$ in $\Delta$,

$$
\phi^{\mu}(A z) A^{\prime}(z)^{2}=\left\{w^{\mu}, A z\right\} A^{\prime}(z)^{2}=\left\{w^{\mu} \circ A, z\right\} .
$$

Therefore, $\phi^{\mu} \in B(G) \cap \Delta$ if and only if for each $A$ in $G$, the restriction of $w^{\mu} \circ A \circ\left(w^{\mu}\right)^{-1}$ to $w^{\mu}\left(U^{*}\right)$ is a linear transformation.

Let $\phi^{\mu}$ belong to $\theta(D(G))$. Let $f=f^{\mu}$ and $w=w^{\mu}$. Let $g$ be the conformal map of $U$ onto $w(U)$ such that $w=g \circ f$. For each $A$ in $G$ there is a conformal map $A_{1}: U \rightarrow U$ which agrees with $f \circ A \circ f^{-1}$ on the real axis. We put $S$ equal to $w \circ A \circ w^{-1}$ in $w\left(U^{*}\right)$ and to $g \circ A_{1} \circ g^{-1}$ in the closure of $w(U) . S$ is quasiconformal everywhere and conformal off $w(R)$. Hence $S$ is everywhere conformal, and $\phi^{\mu} \in B(G) \cap \Delta$.

Conversely, suppose $\phi^{\mu} \in B(G) \cap \Delta$. Let $w=w^{\mu}, f=f^{\mu}$, and $g=w \circ f^{-1}$. Given $A$ in $G$, let $S$ be the linear transformation which agrees with $w \circ A \circ w^{-1}$ in $w\left(U^{*}\right)$. By continuity, $S \circ w=w \circ A$ on the real axis. Therefore, $f \circ A \circ f^{-1}=g^{-1} \circ S \circ g$ on $R$, and the boundary function $h$ of $f$ belongs to $D(G)$. But $\theta(h)=\phi^{\mu}$. Q.E.D.

3. Proof of Theorem 2. Let $\phi_{0}=\phi^{\mu}$ be a point of $\Delta$. We must show that every neighborhood of $\mu$ covers a neighborhood of $\phi_{0}$. Ahlfors [1] proves that if $\left\|\phi-\phi_{0}\right\|$ is sufficiently small, $\phi$ belongs to $\Delta$. With Ahlfors, we write $\phi=\left\{w^{\nu}, z\right\}$ where $w^{\nu}=\hat{f} \circ w^{\mu}$. It suffices to prove that the complex dilatation of $\hat{f}$ tends to zero with $\left\|\phi-\phi_{0}\right\|$.

According to $[1$, p. 300], $\hat{f}$ is the limit of a sequence of mappings $\hat{f}_{n}$. From formula (13) of [1] and the chain rule, we compute that the complex dilatation $\rho_{n}$ of $\hat{f}_{n}$ satisfies

$$
\left\|\rho_{n}\right\|_{\infty}<\frac{\left\|\phi-\phi_{0}\right\|}{\delta-\left\|\phi-\phi_{0}\right\|}
$$


where $\delta$ is a positive constant depending only on $\mu$. Obviously, $\left\|\rho_{n}\right\|_{\infty}$ tends to zero with $\left\|\phi-\phi_{0}\right\|$. Q.E.D.

4. Proof of Theorem 3. We show first that $\theta(T(G))$ contains a neighborhood of the origin in $B(G)$. It is well known [1, pp. 297-299] that every $\phi$ in $B$ with $\|\phi\|<2$ has the form $\phi^{\mu}$ for

$$
\mu(z)=\frac{1}{2}\left(z-z^{*}\right)^{2} \phi\left(z^{*}\right) .
$$

Moreover, it is a simple consequence of the chain rule that $f^{\mu}$ is compatible with $G$ if and only if

$$
\mu(A z)=\mu(z) A^{\prime}(z) / A^{\prime}(z)^{*} \quad \text { for all } A \text { in } G .
$$

If $\phi \in B(G)$ and $\|\phi\|<2$, the $\mu$ in (2) satisfies (3). Hence, $\theta(T(G)$ ) contains the open unit ball in $B(G)$.

Now let $f^{\nu}$ be any mapping compatible with $G$ and let $G^{\nu}$ be the Fuchsian group $f^{\nu} \circ G \circ\left(f^{\nu}\right)^{-1}$. Let $\alpha: T \rightarrow T$ be the right translation which carries the boundary mapping of $f^{\nu}$ to the identity. It is obvious that $\alpha$ maps $T(G)$ onto $T\left(G^{v}\right)$ and $D(G)$ onto $D\left(G^{v}\right)$. Let $\beta: \Delta \rightarrow \Delta$ be the homeomorphism $\theta \circ \alpha \circ \theta^{-1}$. By the Lemma, $\beta$ maps the open set $B(G) \cap \Delta$ in $B(G)$ onto the open set $B\left(G^{v}\right) \cap \Delta$ in $B\left(G^{v}\right)$. Moreover, $\beta$ maps $\phi^{\text {p }}$ to zero.

We have seen that $\theta\left(T\left(G^{v}\right)\right)$ contains the open unit ball $N$ in $B\left(G^{v}\right)$. Since $\alpha$ maps $T(G)$ on $T\left(G^{v}\right), \beta^{-1}(N)$ is contained in $\theta(T(G))$. Since $\beta$ is a homeomorphism of $B(G) \cap \Delta$ on $B\left(G^{v}\right) \cap \Delta, \beta^{-1}(N)$ is open in $B(G)$. Therefore, $\theta(T(G))$ contains a neighborhood of $\phi^{v}$ in $B(G)$. Since $f^{v}$ was any mapping compatible with $G, \theta(T(G))$ is an open set. Q.E.D.

\section{REFERENCES}

1. L. V. Ahlfors, Quasiconformal reflections, Acta Math. 109 (1963), 291-301.

2. L. Bers, Automorphic forms and general Teichmuiller spaces, Proceedings of the Conference on Complex Analysis, Univ. of Minnesota, 1964 (to appear).

3. Z. Nehari, The Schwarzian derivative and schlicht functions, Bull. Amer. Math. Soc. 55 (1949), 545-551.

INSTITUTE FOR AdVANCED STUdY 